\title{
Choreoathetosis - an unusual adverse effect of dihydroartemisinin-piperaquine: a case report
}

\author{
Benjamin Momo Kadia ${ }^{1 *}$, Christian Morfaw ${ }^{2}$ and Armelle Corrine Gounoue Simo 3,4
}

\begin{abstract}
Background: Dihydroartemisinin-piperaquine is a combination of dihydroartemisinin and piperaquine which is highly effective in the treatment of uncomplicated falciparum malaria. Its adverse effects are generally tolerable and temporary. Choreoathetosis, an involuntary movement disorder characterized by continuous irregular twisting of the body, is not a documented adverse effect of this medication.

Case presentation: A 41-year-old Cameroonian man of black African ethnicity was brought to our primary care hospital because over the previous 6 hours he had been experiencing involuntary twisting movements of his body and he no longer had control of his limbs. Earlier that day, he had been prescribed an appropriate dose of dihydroartemisininpiperaquine in our hospital. The abnormal movements started approximately 3 hours after ingesting the first dose of the drug. The review of systems and his past history were unremarkable. On clinical examination, he was conscious and oriented but was unsteady and displayed continuous generalized irregular twisting movements combined with abrupt low amplitude flinging of his limbs. Dihydroartemisinin-piperaquine-induced generalized choreoathetosis was diagnosed. He was sedated with diazepam and dihydroartemisinin-piperaquine was discontinued. The antimalarial drug was substituted with artemether-lumefantrine combination. The clinical progress was good and he was discharged home after 72 hours. No further abnormalities were noted during 7 months of follow-up.
\end{abstract}

Conclusion: Although dihydroartemisinin-piperaquine is increasingly popular as a well-tolerated/efficacious antimalarial drug, clinicians must note the rare possibility of choreoathetosis as an adverse effect of this medication and educate patients accordingly.

Keywords: Dihydroartemisinin-piperaquine, Choreoathetosis, Case report

\section{Background}

Choreoathetosis is an involuntary movement disorder characterized by continuous, non-rhythmic, irregular throwing and writhing of the body which can be focal or generalized. It has diverse etiologies including genetic and hereditary disorders, cerebrovascular disease, poststreptococcal infection, connective tissue diseases, thyroid disorders, hyperglycemia, and post-traumatic brain injury [1]. With regards to pharmacologic agents, neuropsychiatric drugs have been most implicated in the cause of choreoathetosis. Very few antimicrobials have been associated with the onset of this movement disorder.
Dihydroartemisinin-piperaquine (DHA-PPQ) is a fixeddose combination of dihydroartemisinin (DHA) $40 \mathrm{mg}$ and piperaquine (PPQ) $320 \mathrm{mg}$ which is very effective in the treatment of uncomplicated falciparum malaria [2-4]. The reported adverse effects of this drug are generally tolerable and temporary and they include dizziness, headache, cough, nausea, vomiting, anorexia, asthenia, abdominal pain, diarrhea, fever, as well as changes in biochemical and blood indices [5]. This medication can also lead to prolonged QT interval with irregularities in heart rhythm that may be fatal [6]. Choreoathetosis is not a documented side effect of this medication. We, however, report a case of possible DHA-PPQ-associated acute generalized choreoathetosis.

\footnotetext{
* Correspondence: benjaminmomokadia@gmail.com

${ }^{1}$ Foumbot District Hospital, Foumbot, Cameroon

Full list of author information is available at the end of the article
} 


\section{Case presentation}

A 41-year-old Cameroonian man of black African ethnicity was brought to our primary care hospital because over the previous 6 hours he had been experiencing persistent involuntary twisting movements of his body and he no longer had control of his limbs. Earlier that day, he had presented to our hospital with low-grade intermittent fever of nocturnal predominance associated with headache, anorexia, and mild general weakness for 3 days. On that occasion, he was mildly ill-looking and calm. He was alert and oriented in person, place, and time. His vital signs were: temperature, $38.7{ }^{\circ} \mathrm{C}$; blood pressure, 122/83 $\mathrm{mmHg}$; pulse, 91 beats/minute (full and regular); and respiratory rate, 17 breaths/minute. His conjunctivae were pink. His neck was supple. There were no palpable enlarged lymph nodes. His cranial nerve functions as well as deep tendon and cutaneous reflexes were symmetrical and normal. His muscle bulk was good and his muscle tone was normal. Muscle strength was full force. He could execute coordinated movements and he had a normal gait. There was normal sensation to fine touch, crude touch, and vibrations. There was no focal neurological sign and both plantar reflexes were up-going. Chest wall expansions were symmetrical and the cardiac apex beat was located at the left fifth intercostal space in the midclavicular line. Percussion note on his chest wall was resonant. Breath sounds were vesicular and there were no heart murmurs. His abdomen was symmetrical and moved with respiration. There was no abdominal tenderness and no palpable enlarged abdominal organs. The percussion note on his abdomen was tympanic. Bowel sounds were active and normal. The rest of systemic examination was unrevealing as well. Based on those findings, he was presumptively diagnosed with simple malaria. Moderate Plasmodium falciparum parasitemia was found on microscopy (blood film analysis). He was prescribed DHA-PPQ at a dose of $2.1 / 16.8 \mathrm{mg} / \mathrm{kg}$ (total of three tablets of DHA $40 \mathrm{mg}$-PPQ $320 \mathrm{mg}$ ) in a single daily dose and $2 \mathrm{~g}$ of paracetamol tablets in two divided doses per day. These dosages were estimated for his $71 \mathrm{~kg}$ body weight and both drugs were prescribed for 3 consecutive days. Approximately 3 hours after ingesting the first dose of the drug, there was progressive onset of the bizarre movements which were rather amusing to his brother who first thought our patient was joking. However, the movements which were initially intermittent progressively became continuous.

Our patient was not on any other medication prior to the diagnosis of malaria and he had never experienced abnormal movements before. His past history was negative for known chronic illnesses like hypertension, diabetes, neuropsychiatric disorders, chronic kidney disease, or cancer. He had no history of head trauma or neurological disorders like encephalitis or cerebrovascular accident. He had no known history of rheumatic fever. He had no known records of adverse reactions to specific drugs or food and as far as he could remember, he had not ingested DHA-PPQ before. He did not consume tobacco or recreational drugs including traditional medicines. He seldom consumed beer and did not smoke tobacco. He had been a subsistence farmer for 20 years. He lived in the city center and consumed pipeborne water. He had no known history of exposure to environmental hazards such as pesticides, industrial wastes, or other toxic chemicals at home or at work. He was married and had three children. His wife and children were well. He was fifth in a family with four siblings; his siblings and parents were well. His family history was positive for firstdegree relatives with hypertension but negative for movement disorders and other chronic illnesses. There were no known genetic disorders in his family.

On his second visit, a systemic review was unremarkable. His Glasgow Coma Score was 15/15 and he was oriented in person, place, and time. His blood pressure was $133 / 87 \mathrm{mmHg}$, temperature $37.4{ }^{\circ} \mathrm{C}$, and pulse 83 beats/minute. He was very unsteady (making his respiratory rate difficult to appreciate) and displayed continuous irregular twisting movements of his face, neck, extremities, and trunk combined with abrupt forceful low amplitude flinging of his limbs. These movements were precipitated and exacerbated by any voluntary attempts to move such that he could not execute purposeful movements requested by the examiner. Examination of his cranial nerves and grading of muscle strength were difficult because he was unsteady. Muscle tone as well as deep tendon and cutaneous reflexes were normal. The rest of the physical examination was normal.

In view of the historical and clinical data, DHA-PPQassociated acute generalized choreoathetosis was suspected and dystonia was retained as differential diagnosis. Further laboratory investigations such as complete blood count, blood electrolytes, and tests of renal function were without particularities (Table 1). He was sedated with $10 \mathrm{mg}$ of intramuscular diazepam and DHA-PPQ was discontinued. The antimalarial drug was replaced with artemether-lumefantrine combination (20 mg to $120 \mathrm{mg}$ strength - single initial dose of four tablets, followed by four tablets after 8 hours and then four tablets 12 hourly to reach a total of 24 tablets) which was better tolerated. His clinical progress was good and he was discharged home after 72 hours. No further abnormal movements were noted during 3 weeks of regular follow-up. Monthly reviews over the following 7 months were unrevealing.

\section{Discussion}

To the best of our knowledge, this is the first reported case of DHA-PPQ-associated acute generalized 
Table 1 Laboratory test results of the patient

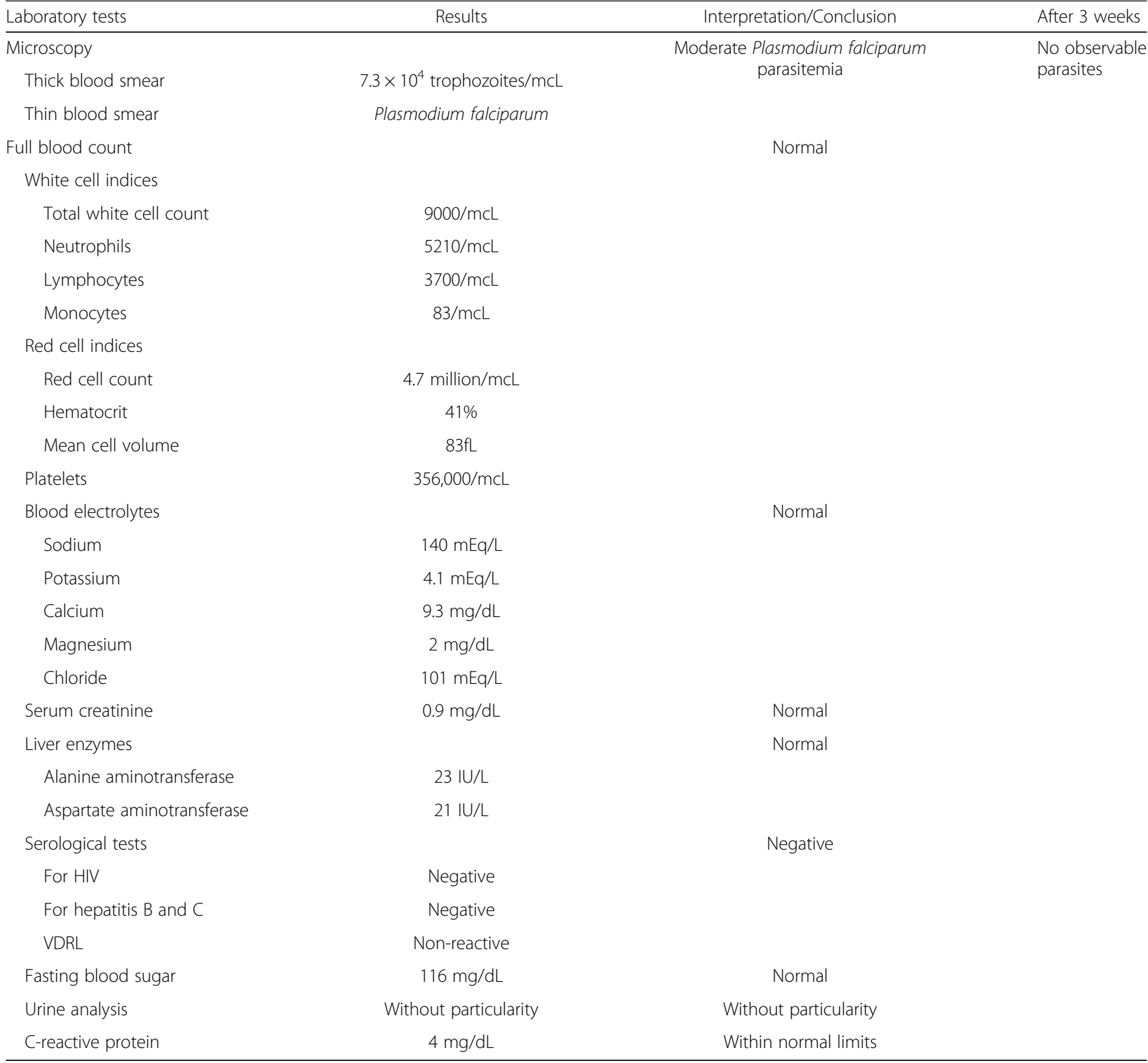

$f L$ femtoliter, $I U / L$ International Units per liter, $m c L$ microliter, $m E q / L$, milliequivalent per liter, $m g / d L$ milligrams per deciliter, VDRL Venereal Disease Research Laboratory

choreoathetosis. Dystonia was considered a differential diagnosis but the swinging of limbs, and the lack of significant abnormalities in posturing/muscle tone made this differential less likely in the case presented. Tardive dyskinesia, rheumatic fever (Sydenham's chorea), and unusual causes of choreoathetosis like endocrine disorders and paraneoplastic syndrome were less plausible etiological differentials given our patient's historical and clinical data, in particular, the temporal relationship between ingestion of DHA-PPQ and onset of choreoathetosis. Furthermore, non-resurgence of the movement disorder after the withdrawal of DHA-PPQ and switching to artemetherlumefantrine lends more credence to a diagnosis of possible DHA-PPQ-induced choreoathetosis. Despite the positive outcome, the case report was flawed by our inability to perform a controlled drug challenge test or measure serum concentrations of DHA-PPQ in order to ascertain that the observed adverse effect was truly unrelated to drug dosage.

Falciparum malaria remains a serious public health concern which is increasingly compounded by the problem of resistance of Plasmodium falciparum to traditional antimalarial drugs like chloroquine and sulfadoxine-pyrimethamine [7]. In order to counter the spread of resistance, the World Health Organization (WHO) recommends the use of artemisinin-based 
combination therapies (ACTs) as first-line treatment of uncomplicated falciparum malaria [8, 9]. ACTs are preparations of short-acting artemisinin derivatives (like artesunate, artemether, and DHA) partnered with longacting drugs. In principle, if a parasite mutation that leads to drug resistance arises during treatment, the parasite should be killed by the long-acting drug, thus limiting the development of resistance [10].

In line with the recent WHO recommendations, various ACTs have been designed and are currently in use on a global scale. One of these is DHA-PPQ. DHA is the active metabolite of the artemisinin derivatives which produces faster relief of clinical symptoms and faster clearance of the parasites from blood when compared with other antimalarial drugs [9]. PPQ, which is a 4-aminoquinoline like chloroquine, has a very long elimination half-life and could provide a long period of posttreatment prophylaxis [11]. Accumulating evidence suggests that the efficacy of DHA-PPQ is comparable to that of commonly used first-line ACTs like artemetherlumefantrine [7, 12].

Although concerns have been raised regarding animal neurotoxicity [13], artemisinin derivatives such as DHA have been used safely in a large number of patients with uncomplicated or severe malaria [14]. PPQ has been used less widely, but studies suggest that it is as potent as chloroquine but less toxic $[15,16]$. However, in a recent randomized controlled trial involving 150 patients, Gargano et al. noted an unusual case of DHAPPQ-induced grand mal convulsions 3 hours after the first appropriate dose of the drug had been administered [17] and this suggests that in rare cases, DHA-PPQ may possibly trigger adverse neurological events. Taking these into account, the unusual incidence of choreoathetosis which we noted in our patient who had ingested an appropriate dose of DHA-PPQ, may suggest an idiosyncratic origin of this movement disorder and possibly other rare neurological complications.

An idiosyncratic drug reaction (IDR) refers to an adverse reaction that does not occur in most patients treated with a drug, does not involve the known pharmacologic properties of that drug, and does not show any apparent dose-response relationship [18]. While noting that IDR characteristically has a delay in onset, rapid onset IDR (as may have been the scenario in our case) is possible [19]. Choreoathetosis in our patient may have been consequent to off-target pharmacology which has been proposed as an idiosyncratic mechanism in many unusual central nervous system adverse drug reactions [18]. In this regard, previous rare reports on drug-induced movement disorders highlight possible roles of imbalanced gamma-aminobutyric acid (GABA) receptor activity, cytokine release, and excessive glutamate which may contribute to altered excitatory and inhibitory cortical and subcortical motor control pathways [20]. Even though these suggestions and the chemical properties of a drug may explain the onset of movement disorders, the underlying patient factors that contribute in individual susceptibility to such rare drug-induced movement disorders are generally poorly elucidated.

\section{Conclusions}

We report a rare case of possible DHA-PPQ-induced choreoathetosis in a black African man who had ingested an appropriate daily dose of the drug. Despite the popular use of DHA-PPQ in the treatment of uncomplicated falciparum malaria and the growing consensus on its efficacy/tolerability, clinicians must note the rare possibility of this central nervous system adverse effect and educate patients accordingly.

\section{Abbreviations}

ACTs: Artemisinin-based combination therapies; DHA: Dihydroartemisinin; DHA-PPQ: Dihydroartemisinin-piperaquine; IDR: Idiosyncratic drug reaction; PPQ: Piperaquine phosphate; WHO: World Health Organization

\section{Acknowledgements}

We thank the patient for agreeing to the publication of his data.

Funding

This research received no funding support

Availability of data and materials

All the data generated in this case report are included in the article.

Authors' contributions

BMK managed the patient, interpreted his data, and prepared the manuscript. CM edited and critically reviewed the final manuscript. ACGS participated in drafting and reviewing the initial manuscript. All the authors read and approved the final manuscript.

Ethics approval and consent to participate

Not applicable.

\section{Consent for publication}

Written informed consent was obtained from the patient for publication of this case report. A copy of the written consent is available for review by the Editor-in-Chief of this journal.

\section{Competing interests}

The authors declare that they have no competing interests.

\section{Publisher's Note}

Springer Nature remains neutral with regard to jurisdictional claims in published maps and institutional affiliations.

\section{Author details}

${ }^{1}$ Foumbot District Hospital, Foumbot, Cameroon. ${ }^{2}$ West Region Technical Group for the fight against HIV/AIDS, Bafoussam, Cameroon. ${ }^{3}$ Faculty of Health Sciences, University of Buea, Buea, Cameroon. ${ }^{4}$ Clinical Research Education, Networking and Consultancy, Douala, Cameroon.

Received: 17 July 2017 Accepted: 23 November 2017

Published online: 28 December 2017

\section{References}

1. Jankovic A, Lang AE. Diagnosis and assessment of Parkinson's disease and other movement disorders. In: Daroff RB, Lankovic J, Mazziotta JC, Pomeroy SC, editors. Bradley's Neurology in Clinical Practices. 7th ed. London: Elsevier; 2016. p. 223-49. 
2. Denis MB, Davis TM, Hewitt $S$, Incardona $S$, Nimol K, Fandeur T, Poravuth $Y$ Lim C, Socheat D. Efficacy and safety of dihydroartemisinin-piperaquine (Artekin) in Cambodian children and adults with uncomplicated falciparum malaria. Clin Infect Dis. 2002:35:1469-76.

3. Tran TH, Stepniewska K, White NJ, Farrar J. Dihydroartemisinin-Piperaquine against multidrug resistant Plasmodium falciparum malaria in Vietnam: randomised clinical trial. Lancet. 2004;363:18-22.

4. Piola P, Fogg C, Bajunirwe F, Biraro S, Grandesso F, Ruzagira E, Babigumira J, Kigozi I, Kiguli J, Kyomuhendo J, Ferrandini L, Taylor W, Checchi F, Guthmann JP. Supervised versus unsupervised intake of six-dose artemetherlumefantrine for treatment of acute uncomplicated Plasmodium falciparum malaria in Mbarara, Uganda: a randomised trial. Lancet. 2005;365:1467-73.

5. Adam I, Tarning J, Lindegardh N, Mahgoub H, McGready R, Nosten F. Pharmacokinetics of Piperaquine in Pregnant Women in Sudan with Uncomplicated Plasmodium falciparum Malaria. Am J Trop Med Hyg. 2012;87(1):35-40

6. World Health Organization. Malaria Policy Advisory Committee Meeting: The cardiotoxicity of antimalarials 2017. Geneva: World Health Organization; 2017.

7. Yavo W, Faye B, Kuete T, Djohan V, Oga SA, Kassi RR, Diata M, Ama MV, Tine R, N'diaye JL, Evi JB, Same-Ekobo A, Faye O, Koné M. Multricentric assessment of the efficacy and tolerability of dihydroartemisinin-piperaquine compared to artemether-lumefantrine in the treatment of uncomplicated Plasmodium falciparum malaria in sub-Saharan Africa. Malar J. 2011;10:198.

8. World Health Organization. World malaria report 2008. Geneva: World health Organization; 2008.

9. World Health Organization. Global Malaria Programme: Guidelines for treatment of malaria 2010. Geneva: World Health Organization; 2010.

10. White NJ, Ollario PL. Strategies for the prevention of antimalarial drug resistance: rational for combination chemotherapy for malaria. Parasitol Today. 1996;12(10):399-401.

11. Keating GM. Dihydroartemisinin/Piperaquine: a review of its use in the treatment of uncomplicated Plasmodium falciparum malaria. Drugs. 2012;72(7):937-61.

12. Ogutu BR, Onyango KO, Koskei N, Omondi EK, Ongecha JM, Otieno GA, Obonyo C, Otieno L, Eyase F, Johnson JD, Omollo R, Perkins DJ, Akhwale W, Juma E. Efficacy and safety of artemether-lumefantrine and dihydroartemisininpiperaquine in the treatment of uncomplicated Plasmodium falciparum malaria in Kenyan children aged less than five years: results of an open-label, randomized, single-centre study. Malar J. 2014;13:33.

13. Brewer TG, Grate SJ, Peggins JO, Weina PJ, Petras JM, Levine BS, Heiffer MH, Schuster BG. Fatal Neurotoxicity of Arteether and Artemether. Am J Trop Med Hyg. 1994;51:251-9.

14. Hien TT, White NJ. Qinghaosu. Lancet. 1993;341:603-8.

15. Zhang KY, Zhou JX, Wu Z, Huang QL. Susceptibility of Plasmodium falciparum to chloroquine, piperaquine, amodiaquine, mefloquine and quinine with in vitro microtechnique in Hainan Island [in Chinese]. Chin J Parasitol Parasitic Dis. 1987;5:165-9.

16. Lan CX, Lin X, Huang ZS, Chen YS, Guo RN. In vivo sensitivity of Plasmodium falciparum to piperaquine phosphate assayed in Linshui and Baisha Counties, Hainan Province [in Chinese]. Chin J Parasitol Parasitic Dis. 1989:7:163-5.

17. Gargano N, Ubben D, Tommasini S, Bacchieri A, Corsi M, Bhattacharyya PC, Rao BHK, Dubashi N, Dev V, Ghosh SK, Kumar A, Srivastava B, Valecha N. Therapeutic efficacy and safety of dihydroartemisinin-piperaquine versus artesunate-mefloquine in uncomplicated Plasmodium falciparum malaria in India. Malar J. 2012;11:233.

18. Zaccara G, Franciotta D, Perucca E. Idiosyncratic Adverse Reactions to Antiepileptic Drugs. Epilepsia. 2007:48(7):1223-44.

19. Uetrecht J, Naisbitt DJ. Idiosyncratic Adverse Drug Reactions: Current concepts. Pharmacol Rev. 2013:65:779-808.

20. Sathirapanya P. Choreoathetosis Is a Possible Adverse Event of a Commonly Used Antibiotic. Case Rep Neurol. 2017:9:81-5.

\section{Submit your next manuscript to BioMed Central and we will help you at every step:}

- We accept pre-submission inquiries

- Our selector tool helps you to find the most relevant journal

- We provide round the clock customer support

- Convenient online submission

- Thorough peer review

- Inclusion in PubMed and all major indexing services

- Maximum visibility for your research

Submit your manuscript at www.biomedcentral.com/submit
Biomed Central 